\title{
Estimation of salivary arginase levels in smokers and non- smokers with chronic periodontitis: A biochemical study
}

\author{
Shruthi Hegde ${ }^{1 *}$, Cynthia Nunes ${ }^{2}$, Harini $\mathrm{K}^{3}$, Subhas Babu ${ }^{4}$, Sucheta Kumari ${ }^{5}$ and Vidya Ajila ${ }^{1}$ \\ ${ }^{1}$ Department of Oral Medicine and Radiology, A.B Shetty Memorial Institute of Dental Sciences, Nitte University, Mangalore, India \\ ${ }^{2}$ A.B Shetty Memorial Institute of Dental Sciences, Nitte University, Mangalore, India \\ ${ }^{3}$ Department of Periodontics, A.B Shetty Memorial Institute of Dental Sciences, Nitte University, Mangalore, India \\ ${ }^{4}$ Department of Oral Medicine and Radiology, A.B Shetty Memorial Institute of Dental Sciences, Nitte University, Mangalore, India \\ ${ }^{5}$ Department of Biochemistry, K S Hegde Medical Academy, Nitte University, Mangalore, India
}

\begin{abstract}
Background: Chronic Periodontitis is the most frequently occurring form of periodontal diseases. Progression of the chronic periodontitis might lead to both esthetic and functional impairment. Early diagnosis and treatment will prevent its progression. Salivary biomarkers such as arginase activity estimation will be of help for assessing the present periodontal status. In the present study, salivary arginase activity was assessed in healthy subjects, in non smokers with chronic periodontitis and in smokers with chronic periodontitis.

Methods: A total of 45 subjects were divided into 3 equal groups such as 15 controls, 15 non smokers with chronic periodontitis and 15 smokers with chronic periodontitis. Periodontal disease status was determined by recording the plaque index (PI), gingival index (GI), Mean probing pocket depth (PPD) and Mean clinical attachment level (CAL). Saliva was collected from patients by spit method. Arginase Activity was estimated by Diacetylmonoxime Method.

Results: The data was analysed using statistical software package R2 O.15 version. The full mouth clinical indices values were analysed by mean and standard deviation, one way ANOVA test. The comparison of the three groups and the each parameter was carried out by post hoc (Tukey). Analysis of salivary arginase levels in various study groups showed the statistical significant difference ( $\mathrm{P}$ value $-<0.001)$ between the control and study groups.

Conclusion: Study showed increased salivary arginase levels in chronic periodontitis patients than healthy controls. Smokers with chronic periodontitis exhibited further increased arginase activity on comparison with non smokers with chronic periodontitis. Study indicated that smoking may have an adverse role on the periodontal status. The present study results suggested that the salivary arginase levels estimation can aid as an easy diagnostic method for assessing the periodontal status.
\end{abstract}

\section{Introduction}

Periodontal diseases are a group of conditions affecting the supporting structures of the dentition. Chronic periodontitis is the most frequently occurring form of periodontal disease. It is characterized by the clinical attachment loss, alveolar bone loss, periodontal pockets, gingival recession, and gingival inflammation. In addition to this drifting of teeth, teeth mobility, and tooth exfoliation may occur, which will lead to aesthetic and functional impairment [1]. Studies have provided support that periodontitis, may confer independent risks for different systemic conditions like osteoporosis, diabetes mellitus, pulmonary infections, pre-term low-weight births and cardiovascular diseases [2].

Periodontal diseases have multifaceted modes of pathogenesis. Periodontitis is initiated by a bacterial biofilm accumulating on the surfaces of the teeth, leading to an excessive inflammatory response [3]. This process is influenced by several risk factors such as stress and/ or host specific factors or habits such as smoking. Host or microbialderived enzymes including arginase, nitric oxide synthase and collagenase are reported to be the cause of destruction of periodontal tissues [4].

Clinical measurements used in the diagnosis of periodontal diseases are often of limited usefulness in that they are indicators of previous periodontal diseases rather than present disease activity [5]. Saliva is appealing for use as a diagnostic fluid for analysis for oral related disease because it is rapid, easy and non-invasive to collect, and generally readily abundant [6,7]. Analysis of saliva may be especially beneficial in the determination of current periodontal status [5].

Arginase is one of the important enzymes found in saliva which is crucially involved in various aspects of inflammation. Increase in the arginase levels leads to increased susceptibility to bacterial infection [1]. Arginase is a semi-essential amino acid involved in various physiological processes such as protein and urea synthesis. It is the fifth enzyme of the urea cycle that hydrolyses arginine to ornithine and urea. Arginase is found mainly in the human liver, however it can also be found in other tissues such as salivary glands [4]. Studies have

Correspondence to: Shruthi Hegde, Department of Oral Medicine and Radiology, A. B Shetty Memorial Institute of Dental Sciences, Nitte University, Mangalore, India, E-mail: drshruthihegde@yahoo.co.in

Key words: periodontitis, arginase, smoking

Received: December 07, 2014; Accepted: January 31, 2015; Published: February 07,2015 
been conducted to examine the efficacy of salivary enzymes activity as a marker of periodontal disease process and to predict the disease progression. Recent studies have demonstrated a higher arginase activity in patients with chronic periodontitis compared to healthy control subjects [5]. Effect of smoking on periodontal status was also evaluated in recent years. It has been reported that smoking favours the arginase pathway, which may be attributable to different components of cigarette smoke (nicotine) [8]. Previous studies have shown that smokers exhibit greater disease level than non-smokers [9]. In view of this data, the aim of our study is to evaluate and compare the salivary arginase levels in healthy individuals, in non-smokers with chronic periodontitis and smokers with chronic periodontitis.

\section{Materials and methods}

The present study included 45 individuals visiting the Department of Oral Medicine and Radiology of A.B. Shetty Memorial Institute of Dental Sciences, Mangalore, India. This study was approved by the ethical committee of the institution. Informed consent was obtained from the subjects volunteered to participate in the study after a detailed explanation of the procedures and objectives of the study. Data regarding the personal history, medical, dental, habit history was recorded. After screening, the patients were selected for the study. Based on the selection criteria mentioned below, they were divided in 3 groups. Patients included in the study were aged between 18 to 60 years and were systemically healthy. Criteria for test group and control group were as follows.

Group A (Control): 15 systemically and Periodontally healthy individuals (nonsmokers without periodontitis) with probing depth $<3 \mathrm{~mm}$ and with no clinical attachment loss.

Group B (Test group): 15 Nonsmokers with chronic periodontitis. Patients having probing depth $\geq 5 \mathrm{~mm}$ and clinical attachment loss.

Group C (Test Group):15 Smokers with chronic periodontitis. Patients with probing depth $\geq 5 \mathrm{~mm}$, clinical attachment loss and smoking history of smoking more than ten cigarettes per day for more than ten years

Subjects with any chronic illness, on any medication from last 6 months, patients who have undergone or undergoing any periodontal therapy were excluded from the study.

\section{Determination of periodontal disease status}

Periodontal examination was done by a trained examiner. Periodontal disease status was determined by recording the plaque index (PI) by Silness and Loe, gingival index (GI), Mean probing pocket depth (PPD) and Mean clinical attachment level (CAL). PD and CAL were recorded to the nearest millimetre using the Williams graduated periodontal probe at four sites around each tooth (mesiobuccal, midbuccal,distobuccal and midlingual), excluding the third molars. One calibrated examiner obtained all the measurements so as to reduce intra-examiner variability.

\section{Collection of saliva samples}

Saliva was collected from patients by spit method. Early morning samples were collected and centrifuged at 10,000X g for 10 minutes at 4 degrees Celsius and the supernatants were stored immediately at -20 degrees Celsius to be used later to determine the arginase activity

\section{Arginase Activity estimation by Diacetylmonoxime Method}

The Diaacetylomonoxime method is used for the qualitative determination of arginase is spectrophotometric measurement of urea produced by the hydrolysis of L-arginineby arginase. Diacetylmonoxime reacts with urea formed by arginase action under strongly acidic conditions in the presence of ferric ion and thiosemicarbazide gives a pink coloured diacetyle complex which is red spectrophotometrically at $540 \mathrm{~nm}$ and used for the calculation which was directly proportional to the arginase activity.

\section{Results}

The sample size of this study was 45 , which were divided into three equal groups i.e. group A, being the control (n-15), group B -non smokers with chronic periodontitis (n-15), group C- smokers with chronic periodontitis $(n-15)$. The mean age of the subjects was 40.31 \pm 10.148 years. The average of the full mouth clinical parameters i.e. Plaque index (PI), Gingival index (GI), Mean probing depth (PPD) and Mean clinical attachment loss (CAL) were measured. The levels of salivary arginase were calculated for all subjects. The data was analysed using statistical software package $\mathrm{R} 2 \mathrm{O} .15$ version. The $\mathrm{P}$-value less than 0.05 was taken as significant. The measurements of full mouth clinical indices including mean and standard deviation values are summarized in Table 1. The clinical parameters were analysed using one way ANOVA test. In between comparison of the three groups and the each parameter was carried out by post hoc (Tukey) which is summarized in Table 2 . The salivary arginase levels in various study groups are shown in Table 3 and Figure 1. Clinical parameters such as PI, GI, CAL, PPD were compared between the groups. All the parameters are statistically significant $(P$ value $<0.05)$.

Post hoc tukey test analysis was done to determine the statistical significance between the subgroups. The comparison of plaque index between group A and group B showed no statistically significant differences ( $P$ value - 0.635), whereas between group B and group $C$ statistical significant difference was observed $(P$ value -0.001$)$. Similarly the comparison of gingival index in group A and group B showed statistical significance ( $P$ value -0.001$)$. Group $C$ and group A comparison also showed statistically significant value (P value $-<0.001$ ). However group B and group C did not show statistically significant difference $(P$ value - 0.885). These results indicate a much higher GI score in diseased patients when compared with controls. Mean probing pocket depth values when compared between the three groups (group A, group B, group C) showed statistically significant values. In the case of mean clinical attachment level, group A and group B comparison as well as group $\mathrm{A}$ and group $\mathrm{C}$ comparison revealed statistical significant difference $(P$ value $-<0.001)$. However values in group $\mathrm{B}$ and group $\mathrm{C}$ were not found to be statistical significant $(P$ value -0.201$)$.

Analysis of salivary arginase levels in various study groups showed the statistical significant difference $(P$ value $-<0.001)$ between each group (Table 3).

\section{Discussion}

Chronic periodontitis is most frequently occurring infectious inflammatory disease affecting the tooth supported structures. Periodontitis is initiated by bacterial biofilm accumulating on the surface of teeth [1]. Diagnosis of periodontitis is done by examination of the oral cavity and measuring the clinical parameters. Saliva is an important biologic material for diagnosis periodontal diseases [10]. Saliva contains local and systemic derived markers of periodontal disease [1]. A biomarker is defined as a pharmacological or physiological measurement that is used to predict a toxic event; a specific molecule in the body, which has a particular feature that makes it instrumental for 
Table 1. Full mouth clinical indices in three study groups.

\begin{tabular}{|l|c|c|c|c|}
\hline \multicolumn{1}{|c|}{} & Group A & Group B & Group C & Anova Test \\
\hline Plaque index & $1.027333 \pm 0.315741$ & $1.11 \pm 0.134854$ & $1.386 \pm 0.257316$ & 24.526 \\
\hline Gingival index & $0.285333 \pm 0.259886$ & $0.748 \pm 0.342537$ & $0.804 \pm 0.362586$ & 11.527 \\
\hline Mean probing pocket depth (mm) & $1.218 \pm 0.319357$ & $2.672 \pm 0.779443$ & $3.368667 \pm 0.86743$ & 23.102 \\
\hline Mean clinical attachment level (mm) & $0 \pm 0$ & $1.859333 \pm 0.917228$ & $2.304667 \pm 0.79003$ & $<0.001$ \\
\hline
\end{tabular}

Data expressed as mean \pm standard deviation, $P$ value $<0.05$ is statistically significant, GROUP A-control, GROUP B- non smokers with chronic periodontitis, GROUP C- smokers with chronic periodontitis

\begin{tabular}{|c|c|c|c|c|}
\hline Dependent Variable & Group I & Group J & Mean difference (I-J) & $P$ Value \\
\hline \multirow{3}{*}{ Plaque index } & \multirow{2}{*}{ Group A } & Group B & -0.08267 & 0.635 \\
\hline & & Group C & -0.35867 & 0.001 \\
\hline & Group B & Group C & -0.276 & 0.011 \\
\hline \multirow{3}{*}{ Gingival index } & \multirow{2}{*}{ Group A } & Group B & -0.46267 & 0.001 \\
\hline & & Group C & -0.51867 & $<0.001$ \\
\hline & Group B & Group C & -0.056 & 0.885 \\
\hline \multirow{3}{*}{$\begin{array}{l}\text { Mean Probing pocket } \\
\text { depth }\end{array}$} & \multirow{2}{*}{ Group A } & Group B & -1.454 & $<0.001$ \\
\hline & & Group C & -2.15067 & $<0.001$ \\
\hline & Group B & Group C & -0.69667 & 0.024 \\
\hline \multirow{3}{*}{$\begin{array}{l}\text { Mean clinical } \\
\text { attachment level }\end{array}$} & \multirow{2}{*}{ Group A } & Group B & -1.85933 & $<0.001$ \\
\hline & & Group C & -2.30467 & $<0.001$ \\
\hline & Group B & Group C & -0.44533 & 0.201 \\
\hline \multirow{3}{*}{ Salivary arginase levels } & \multirow{2}{*}{ Group A } & Group B & -4.622 & $<0.001$ \\
\hline & & Group C & -9.30933 & $<0.001$ \\
\hline & Group B & Group C & -4.68733 & $<0.001$ \\
\hline
\end{tabular}

$P$ value $<0.05$ is statistically significant, GROUP A-control, GROUP B- non smokers with chronic periodontitis, GROUP C- smokers with chronic periodontitis.

Table 3. Salivary arginase levels (IU/ml) in different study groups.

\begin{tabular}{|l|c|c|c|c|}
\hline & Group A & Group B & Group C & P Value \\
\hline Salivary arginase & $14.45467 \pm 1.68783$ & $19.07667 \pm 1.214964$ & $23.764 \pm 1.12573$ & $<0.001$ \\
\hline
\end{tabular}

Data expressed as mean \pm standard deviation, $P$ value $<0.05$ is statistically significant.

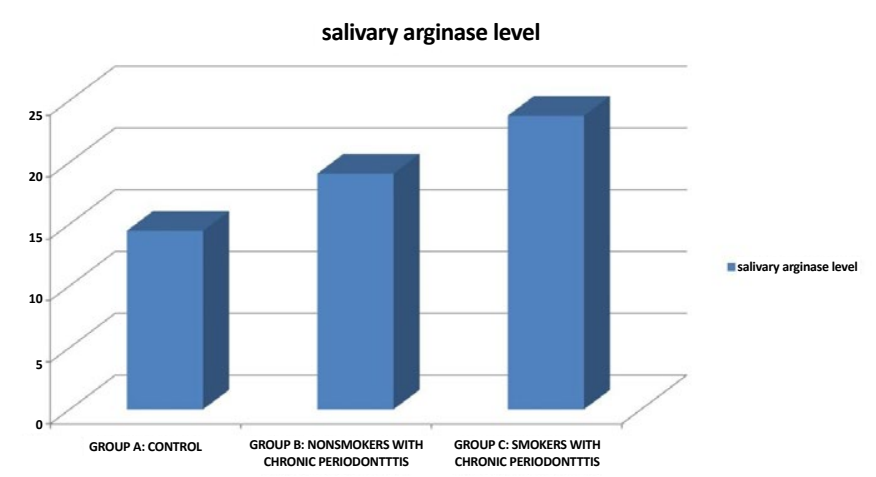

Figure 1. Salivary arginase levels in control and study groups.

measuring disease progression or the effects of treatment. Biomarkers are by definition suitable to develop new diagnostic tools, alone or in combination with traditional methods [11]. Saliva collection is noninvasive and thus is well accepted by patients [3]. Clinical measurements used in the diagnosis are indicators of previous periodontal disease [5]. Analysis of saliva may be beneficial in determination of current periodontal status [12].

Salivary enzymes activity might be a marker to reflect the extent of periodontal destruction [5]. In the previous studies it was believed that an increased arginase activity may lead to less nitric oxide production, which consequently increases the susceptibility of bacterial infection [8]. Previous researchers have showed that smoking may alter the arginase activity. The present study evaluated the salivary arginase levels in healthy individuals, non smokers with chronic periodontitis and smokers with chronic periodontitis.

Arginase and nitric oxide synthetase (NOS) compete for a common substrate, L- arginine. Arginase uses L - arginine to produce urea and ornithine, whereas NOS uses L- arginine to produce nitric oxide. The urea cycle is an essential metabolic pathway due to conversion of ammonia ions into urea. Urea cycle is essential for humans for removal of toxic products. Arginase is one of the five key enzymes of urea cycle $[1,13,14]$.

Nitric oxide is a free radical, produced by L- arginine. Nitric oxide produced may act as cytotoxic molecules against cells infected by bacteria, fungi, protozoa and tumour cells. It may lead to tissue destruction. Due to its antimicrobial activity, it is one of the important molecules against the some infections. Since L- arginine is a common substrate by both arginase and NOS, it is believed that an increased arginase production lead to reduction in nitric oxide production, which in turn lead to increased susceptibility to bacterial infection $[1,13]$. Our study results also demonstrated the higher salivary arginase levels in case of chronic periodontitis patients. This is in accordance with the above mentioned hypothesis.

In the present study salivary arginase levels were increased in chronic periodontitis patients compared to the healthy controls. Further increased values were noted in chronic periodontitis patients with smoking habit. This clearly indicated that salivary arginase levels are increased in the disease group. Statistically significant difference between control group and study groups were noted in our study. Gheren et al. [1] study also revealed similar results. In their study SAA was increased in chronic periodontitis patients compared to the controls. Our results were comparable with the Ozmeric et al. [5] study, in which a significant increase in the salivary arginase levels was noted in periodontitis patients compared to the controls. Salivary arginase activity was assessed before and after nonsurgical periodontal therapy in a study, which showed reduction of salivary arginase activity following therapy [15].

The statistically significant increased salivary arginase levels in smokers with chronic periodontitis were noticed in our study, which suggests that smoking increases salivary arginase activity. This was in accordance with Queiroz et al. [8] study, where salivary arginase levels were measured in smokers and non smokers with and without dental implants. Studies have shown that smoking favours the arginase pathway, which might be attributable to different components of cigarette smoke, especially nicotine [15]. A study conducted in the year 2000 proved that smoking is a major risk factor for periodontitis [9]. Our study also showed similar findings.

Saliva and gingival biopsy samples were compared in a study to examine the arginase nitric oxide-polyamine pathway alteration in patients with gingivitis or periodontitis and healthy controls. They found that gingival tissue samples were more informative about 
periodontal pathogenesis than saliva [4]. This is due to the fact that the composition of saliva is very complex. Saliva samples may also contain blood, gingival crevicular fluid, and extrinsic substances such as food debris, bacteria and bacterial products. Therefore arginase levels in saliva might be affected by various intrinsic, extrinsic factors. In the present study saliva samples were collected, because our aim was to evaluate a non invasive and easy method to assess the periodontal status. In case of gingival tissue samples biopsy is required. Patient acceptance level for saliva sample collection is much higher compared to the gingival biopsy. Saliva samples were examined by various researchers and found valuable for assessing periodontal status $[1,5,15]$.

The periodontal clinical measurements (plaque index, gingival index, mean probing pocket depth, mean clinical attachment level) were recorded in our study. The comparison of plaque index between control group and non-smokers with chronic periodontitis and also smokers with chronic periodontitis was done. Statistical significant difference was observed between control and periodontitis group. The gingival index comparison between controls and chronic periodontitis patients showed statistical significance. However there was no significant difference between non-smokers and smokers with chronic periodontitis. These findings were similar to the Kolte et al. [16] study.

In our study mean probing pocket depth values were compared between the three groups (Group A, Group B, Group C) which showed statistically significant values. Results indicate that the increased probing pocket depth in chronic periodontitis patients compared to normal. Further increase is noted in the smokers.

Mean clinical attachment levels comparison between Group A and Group B as well as group A and group C revealed statistical significant difference. The values of Group B and Group C were statistical insignificant. This was in contrary to findings of Kolte et al. [16] study, where no significant difference was noted.

To the best our knowledge this is the first study conducted in India to estimate the salivary arginase levels in healthy patients, chronic periodontitis patients without smoking habit and with smoking habit. In our study both the clinical parameter and biochemical parameter such as salivary arginase levels was estimated to assess the periodontal status. A significant higher value was noted in clinical parameters as well as salivary arginase levels in chronic periodontitis patients when compared to the healthy persons. Further significantly higher periodontal indices and salivary arginase levels was found in smokers with chronic periodontitis when compared to non-smokers with chronic periodontitis. Results of our study indicate that salivary arginase estimation can be used as a salivary marker to periodontal status.

\section{Conclusion}

Chronic periodontitis is one of the most commonly encountered oral diseases with significant effects on oral and systemic health. Early evaluation of the periodontal status is essential to prevent the progression of the disease. Our study results showed increased salivary arginase levels in chronic periodontitis patients as compared to healthy controls. Also, among patients with chronic periodontitis, smokers exhibited further increased arginase activity in comparison to non smokers. Thus our study implies that salivary arginase levels could be used as a reliable marker of the periodontal status of an individual.

\section{References}

1. Gheren LW, Cortelli JR, Rodrigues E, Holzhausen M, Saad WA (2008) Periodontal therapy reduces arginase activity in saliva of patients with chronic periodontitis. Clin Oral Investig 12: 67-72. [Crossref]

2. Meurman JH, Sanz M, Janket SJ (2004) Oral health, atherosclerosis, and cardiovascular disease. Crit Rev Oral Biol Med 15: 403-413. [Crossref]

3. Bertl K, Haririan H, Laky M, Matejka M, Andrukhov O, et al. (2012) Smoking influences salivary histamine levels in periodontal disease. Oral Dis 18: 410-416. [Crossref]

4. Ozer L, Elgun S, Ozdemir B, Pervane B, Ozmeric N (2011) Arginine-Nitric OxidePolyamine Metabolism in Periodontal Disease. J Periodontol 82: 320-8.

5. Ozmeriç N, Elgün S, Uraz A (2000) Salivary arginase in patients with adult periodontitis Clin Oral Investig 4: 21-24. [Crossref]

6. Lee YH, Wong DT (2009) Saliva: an emerging biofluid for early detection of diseases Am J Dent 22: 241-248. [Crossref]

7. Malamud D (2011) Saliva as a diagnostic fluid. Dent Clin North Am 55: 159-178. [Crossref]

8. Queiroz DA, Cortelli JR, Holzhausen M, Rodrigues E, Aquino DR, et al. (2009) Smoking increases salivary arginase activity in patients with dental implants. Clin Oral Investig 13: 263-267. [Crossref]

9. Tomar SL, Asma S (2000) Smoking-attributable periodontitis in the United States findings from NHANES III. National Health and Nutrition Examination Survey. $J$ Periodontol 71: 743-751. [Crossref]

10. Khashu H, Baiju CS, Bansal SR, Chillar A (2012) Salivary Biomarkers: A Periodontal Overview. J Oral Health Comm Dent 6: 28-33.

11. Al-Tarawneh SK, Border MB, Dibble CF, Bencharit S (2011) Defining salivary biomarkers using mass spectrometry-based proteomics: a systematic review. OMICS 15: 353-361. [Crossref]

12. Zia A, Khan S, Bey A, Gupta ND, Mukhtar-Un-Nisar S (2011) Oral biomarkers in the diagnosis and progression of periodontal diseases. Biol Med 3: 45-52.

13. Güllü C, Ozmeric N, Tokman B, Elgün S, Balos K (2005) Effectiveness of scaling and root planing versus modified Widman flap on nitric oxide synthase and arginase activity in patients with chronic periodontitis. J Periodontal Res 40: 168-175. [Crossref]

14. Munder M (2009) Arginase: an emerging key player in the mammalian immune system. Br J Pharmacol 158: 638-651. [Crossref]

15. Pereira AL, Cortelli SC, Aquino DR, Franco GC, Cogo K, et al. (2012) Reduction of salivary arginine catabolic activity through periodontal therapy. Quintessence Int 43 : 777-787. [Crossref]

16. Kolte AP, Kolte RA, Laddha RK (2012) Effect of smoking on salivary composition and periodontal status. J Indian Soc Periodontol 16: 350-353. [Crossref]

Copyright: (C2015 Hegde S. This is an open-access article distributed under the terms of the Creative Commons Attribution License, which permits unrestricted use, distribution, and reproduction in any medium, provided the original author and source are credited. 